05

\title{
Экспериментальная оценка структурно-временных характеристик разрушения материала на основе магнитно-импульсного нагружения кольцевых образцов
}

\author{
(С) В.А. Морозов, ${ }^{1}$ Ю.В. Петров, ${ }^{1}$ В.Д. Сухов ${ }^{2}$ \\ ${ }^{1}$ Санкт-Петербургский государственный университет, \\ 198504 Санкт-Петербург, Россия \\ ${ }^{2}$ Университет ИТМО, \\ 197101 Санкт-Петербург, Россия \\ e-mail: vdsukhov@ya.ru, yp@yp1004.spb.edu
}

Поступило в Редакцию 13 октября 2018 г.

В окончательной редакции 13 октября 2018г.

Принято к публикации 29 октября 2018 г.

\begin{abstract}
Проведено исследование разрушения тонких алюминиевых колец при ударном воздействии магнитноимпульсным способом. Данный способ позволяет проводить эксперименты по разрушению образцов в условиях чистого растяжения для широкого диапазона скоростей деформации. Для моделирования разрыва кольцевых образцов применен структурно-временной критерий. Данный критерий позволяет анализировать эффекты, характерные для динамических испытаний. Рассчитаны инкубационное время разрушения алюминиевых колец при магнитно-импульсном нагружении и значения критических напряжений. Получена аналитическая зависимость между критическим напряжением и временем разрушения, которая находится в соответствии с экспериментальными данными.
\end{abstract}

DOI: 10.21883/JTF.2019.05.47470.364-18

\section{Введение}

Изучение разрушения материалов при воздействии сверхкоротких нагружающих импульсов играет важную роль в механике деформируемого твердого тела и физике. Результаты таких исследований применяются во множестве прикладных задач, таких как создание различных систем в машиностроении, развитие электрофизических технологий и энергетики.

Эксперименты по высокоскоростному разрушению демонстрируют разительные отличия от аналогичных экспериментов при квазистатических воздействиях. Так, например, при высокоскоростном нагружении ярко выражена зависимость критических значений напряжений от истории и методов нагружения. Данная зависимость предельных величин проявляется при изменении длительности воздействия, скорости роста внешнего воздействия, а также множества других причин. В случае медленного нагружения критическое напряжение, вызывающее разрушение материала, можно считать константой. Такой подход перестает работать, как только рассматриваются достаточно короткие временные профили напряжений, так как предельные значения напряжений начинают проявлять ярко выраженную зависимость от длительности воздействия.

В настоящее время множество опытов по динамическому нагружению реализуют с помощью воздействия на образец ударника. В результате такого воздействия в образце возникает волна сжатия, которая, отражаясь от свободной поверхности, преобразуется в волну растяжения. Комбинация волн способна вызвать разрыв материала, как это случается в традиционных экспериментах на откол. Отмечается, что предварительная стадия интенсивного сжатия способна изменять структуру рассматриваемого материала. Поэтому необходимо развитие новых подходов, которые позволят проводить эксперименты по непосредственному растяжению в условиях импульсного воздействия. Такими свойствами обладают магнитно-импульсные методы.

В настоящей работе рассматривается сверхбыстрый разрыв тонких колец, выполненных из конденсаторных алюминиевых фольг. С помощью разработанных магнитно-импульсных методов [1-5] получен набор экспериментальных данных, который позволил построить временную зависимость прочности кольцевых образцов в широком диапазоне длительностей воздействия. Построена теоретически рассчитанная зависимость между предельным значением напряжения и временем разрушения. Для моделирования разрушения был использован подход, основанный на применении структурновременного критерия [6,7]. Основываясь на данном подходе, определяются предельные значения, которые характеризуют момент разрыва образца.

\section{Методика эксперимента и результаты}

Для получения данных о разрушении алюминиевых образцов были проведены эксперименты по магнитноимпульсному нагружению с использованием генератора коротких высоковольтных импульсов ГКВИ-300, позволяющего реализовывать различные формы электриче- 

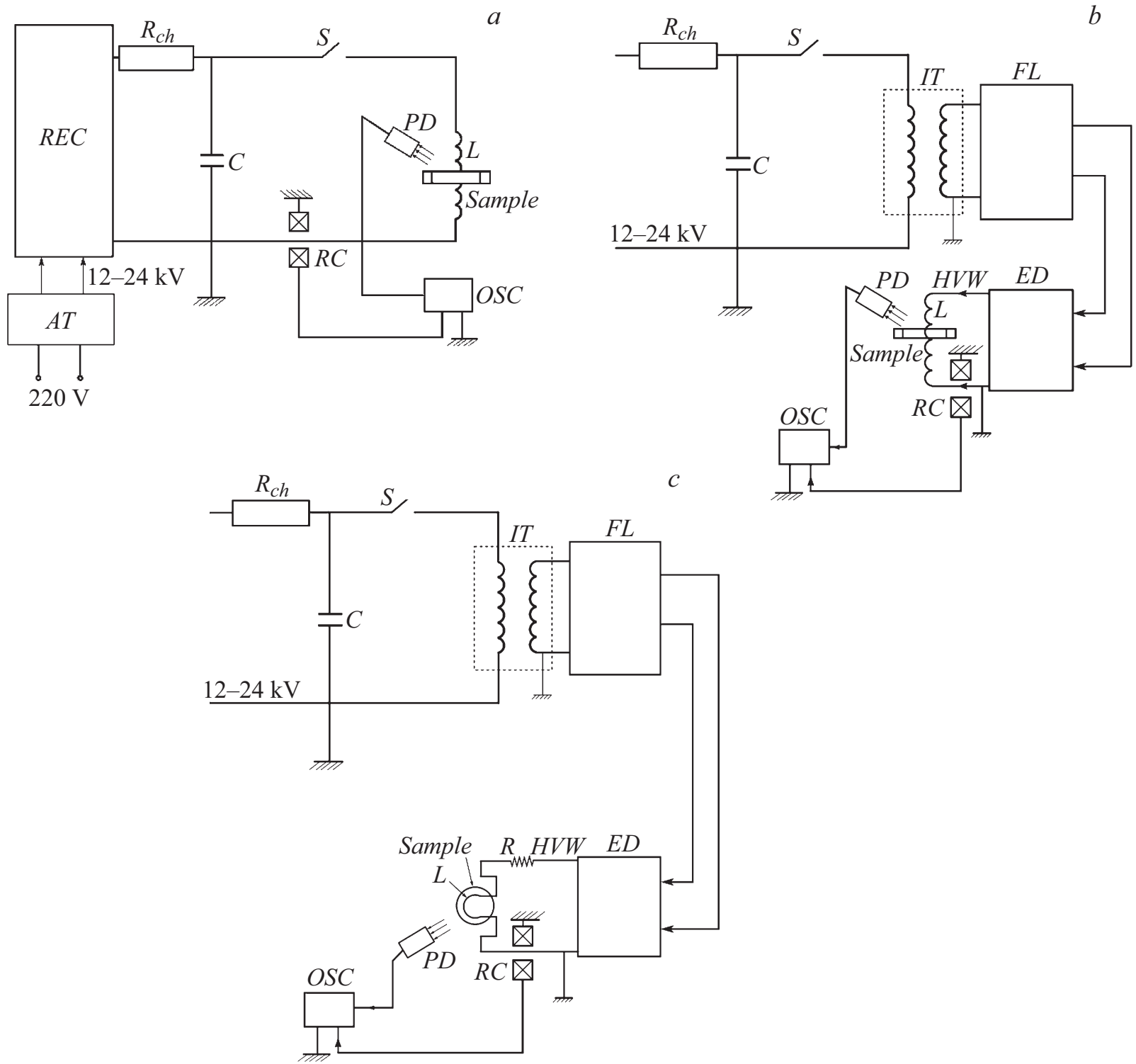

Рис. 1. Блок-схемы нагружения образцов. $A T-$ автотрансформатор, REC - выпрямитель, $R_{c h}-$ зарядное сопротивление, $C$ - емкость, $S-$ разрядник, $R C$ - пояс Роговского, $L-$ катушка, $P D-$ фотодиод, $O S C$ - осциллограф, sample - образец, $I T$ - импульсный трансформатор, $E D$ - выходное устройство, $H V W-$ высоковольтный электрод, $F L-$ формирующая линия.

ских напряжений с амплитудами $30-300 \mathrm{kV}$. Временные профили механических напряжений могут эффективно управляться подбором электрических параметров импульсного генератора. С целью построения временной зависимости разрушения образцов в широком интервале (от 0.3 до $6 \mu \mathrm{s}$ ) были предложены три схемы нагружения (рис. 1).

На рис. 1, $a$ представлена блок-схема первой установки, которая реализует гармоническое нагружение с периодом в интервале 5.5-7.5 $\mu \mathrm{s}$. Кольцевой образец закрепляется соосно с катушкой, по которой проходит ток. Данный ток вызывает в образце индукционный ток. В результате взаимодействия токов в катушке и кольцевом образце возникает сила отталкивания, которая равномерно распределена по внутренней поверхности кольца (рис. 2). Вследствие данной нагрузки в кольце возникают окружные напряжения, которые играют главную роль в разрушении образца. Эксперименты проводились таким образом, чтобы разрыв образца происходил лишь в одном месте.

Для осуществления более скоростных нагружений были предложены две схемы. Первая из них (рис. 1, $b$ ) реализует электромагнитные колебания в катушке индуктивности с периодом $1 \mu \mathrm{s}$. C помощью второй схемы (рис. $1, c)$ осуществляется апериодический режим колебаний, тем самым формируется импульсное воздействие на образцы с длительностью импульса $80 \mathrm{~ns}$.

На рис. 3 приведены фотографии экспериментальных установок для вышеприведенных блок-схем. 


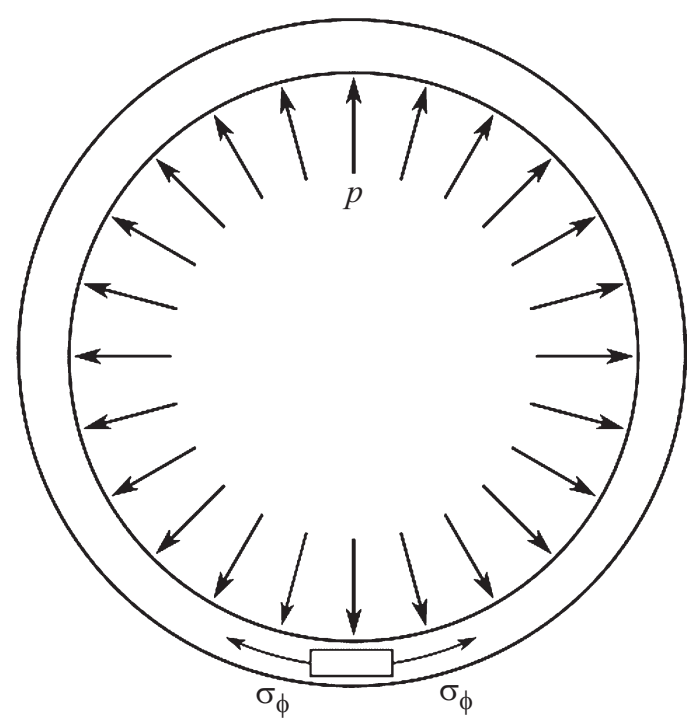

Рис. 2. Схема нагружения образца ( $p-$ нагрузка, $\sigma_{\phi}-$ растягивающее напряжение).
В экспериментах использовались конденсаторные алюминиевые фольги толщиной $15 \mu \mathrm{m}$ и шириной $1-5 \mathrm{~mm}$.

При проведении высокоскоростных экспериментов необходимо уметь точно определять время, соответствующее разрушению образца. Для решения этой проблемы был предложен новый оригинальный подход. Время до момента разрушения определялось по вспышке в месте разрыва образца, которую фиксировал фотодиод (рис. 1). Вспышка образуется вследствие того, что невозможно мгновенно прервать ток, протекающий по образцу. Сигнал с фотодиода синхронизировался с моментом подачи тока на катушку.

Пример образца после разрушения при пороговом значении энергии приведен на рис. 4.

Окружное напряжение разрыва кольцевого образца $\sigma(t)$ определялось по измеренному радиальному давлению $q(t)$ с помощью специально разработанного пьезодатчика по формуле Лапласа: $\sigma(t)=R_{0} q(t) / h$, где

$a$
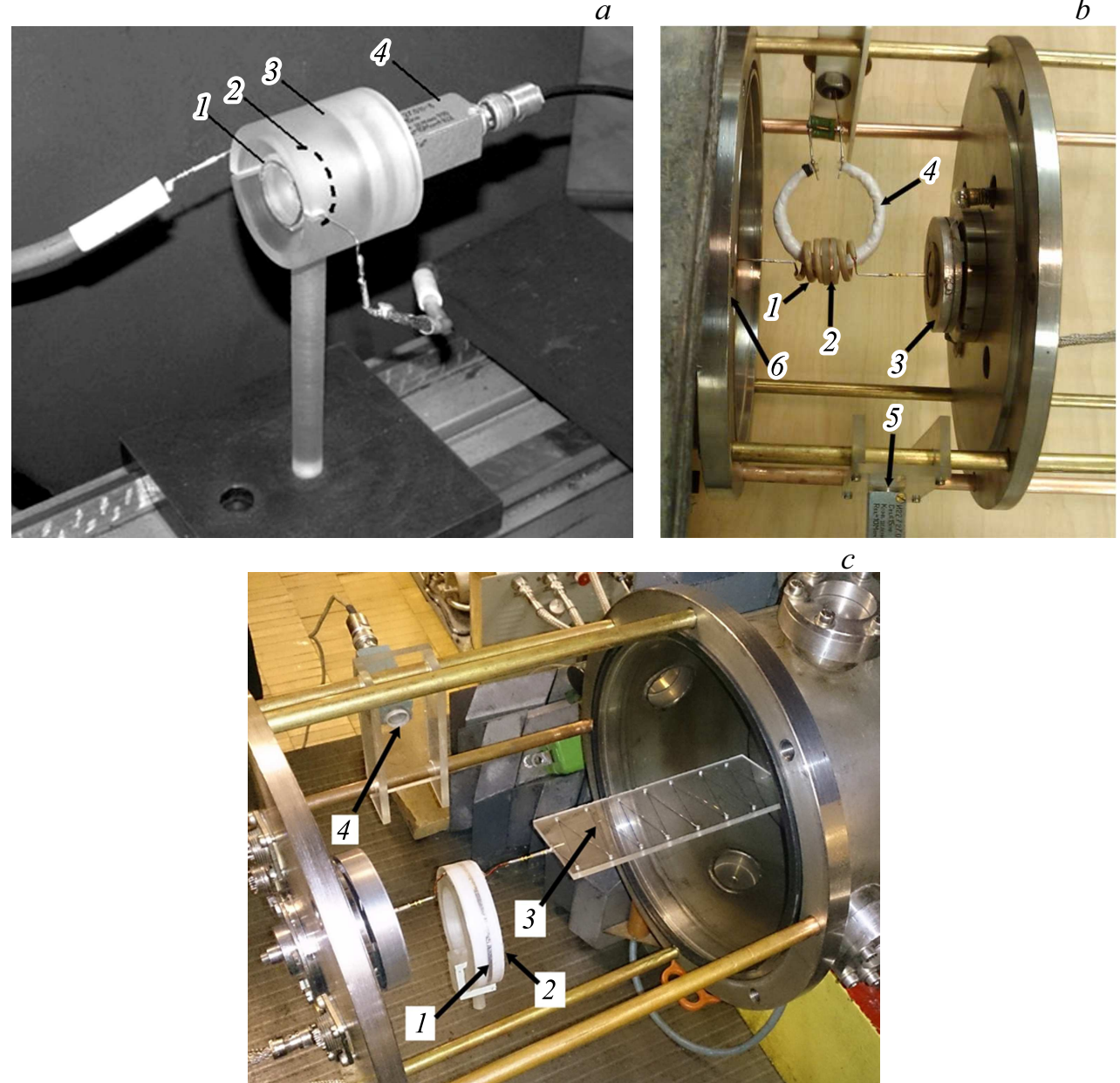

Рис. 3. Внешний вид установок: $a-$ при $T=7.5 \mu \mathrm{s}(1-$ катушка, $2-$ образец, $3-$ корпус, позволяющий улавливать фрагменты разрушенного образца, $4-$ фотодиод); $b-$ при $T=1 \mu \mathrm{s}(1-$ катушка, $2-$ образец, 3 - пояс Роговского, измеряющий ток в катушке, $4-$ пояс Роговского, измеряющий ток в кольце, $5-$ фотодиод, $6-$ выходное устройство $) ; c-$ при $T=80 \mathrm{~ns}(1-$ образец, 2 - виток катушки, 3 - добавочное сопротивление, 4 - фотодиод). 


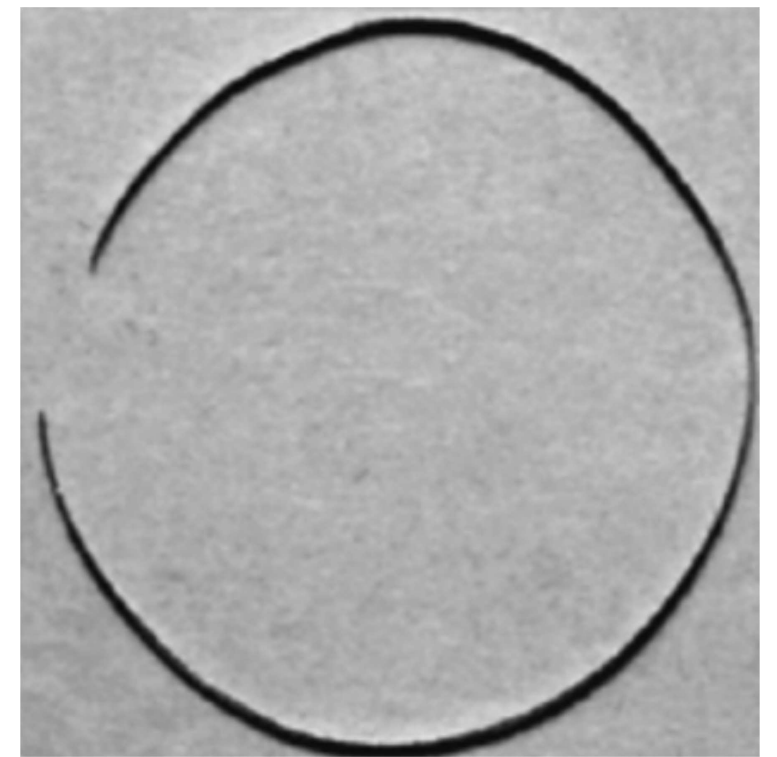

Рис. 4. Фотография разрушенного образца при пороговом значении энергии $(T=7.4 \mu \mathrm{s})$.

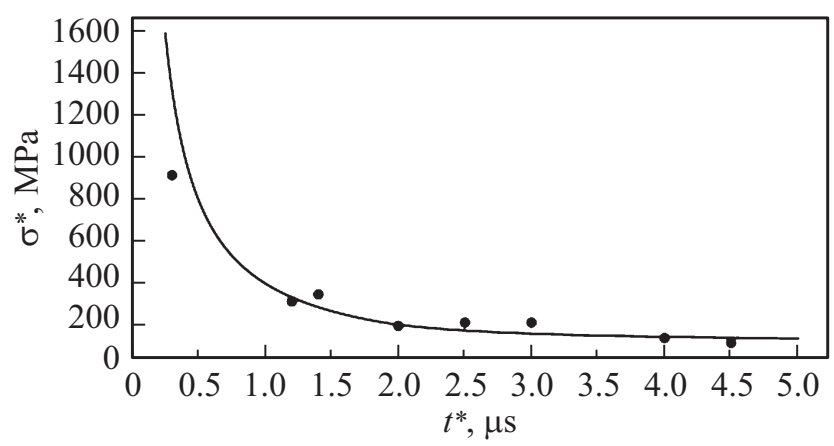

Рис. 5. Зависимость прочности алюминиевых кольцевых образцов от времени разрушения.

$R_{0}$ - начальный радиус кольцевого образца, $h-$ его толщина.

Полученные экспериментальные данные позволили построить кривую временной зависимости прочности кольцевого образца из алюминиевой фольги, представленную на рис. 5. Зависимость можно условно разделить на две „ветви“. Горизонтальная „ветвь“ описывает слабую зависимость критических напряжений от времени разрушения и обычно называется квазистатической. Вертикальная „ветвь“ описывает процесс быстрого разрушения, т. е. при достаточно коротких временах разрушения, и называется динамической. Данные квазистатической ветви разрушения образцов соответствуют экспериментальным данным работы [1], а также данным по статическим испытаниям [2] тех же алюминиевых фольг, которые подвергались динамическому разрушению. Следует также отметить совпадение наших результатов по испытанию образцов в микросекундном диапазоне длительностей разрушения $(\sim 1 \mu \mathrm{s})$ с данными [8].

\section{Критерий разрушения}

В случае рассмотрения квазистатических задач при описании поведения материала можно использовать критерий для предельного значения напряжения $\sigma(t)$ :

$$
\sigma(t) \leqslant \sigma_{c},
$$

где $\sigma_{c}$ - предельное значение напряжения для квазистатических экспериментов по разрушению, $t-$ время. В данном случае можно считать, что $\sigma_{c}$ остается постоянным для всего квазистатического процесса нагружения образца.

С помощью структурно-временного подхода было смоделировано разрушение алюминиевых кольцевых образцов. Данный подход позволяет предсказывать множество эффектов, которые наблюдаются в опытах. Для задачи о разрыве колец структурно- временной подход формулируется следующим образом:

$$
\frac{1}{\tau} \int_{t-\tau}^{t} \sigma(\xi) d \xi \leqslant \sigma_{c},
$$

где $\tau-$ инкубационное время разрушения. Инкубационное время - это параметр, который отвечает за предварительные процессы, протекающие в теле перед образованием макроразрушения. Структурно-временной критерий был предложен в [9]. Время, при котором данное неравенство впервые обращается в равенство, соответствует времени разрушения $t^{*}$. Следует отметить, что при медленном изменении напряжения $\sigma(t)$ критерий (2) переходит в (1).

Предложенный подход также применяется для решения задач электропробоя в диэлектриках, кавитации в сплошных средах, откольного разрушения, движения трещин и т. д. [10-13]. К числу эффектов, которые можно объяснить с помощью структурно-временного подхода, относится явление эффекта запаздывания разрушения. Это явление состоит в том, что разрушение образца может возникать и на участке спада импульса напряжения.

Будем для всех испытаний аппроксимировать импульс напряжений треугольником (рис. 6). Запишем аналити-

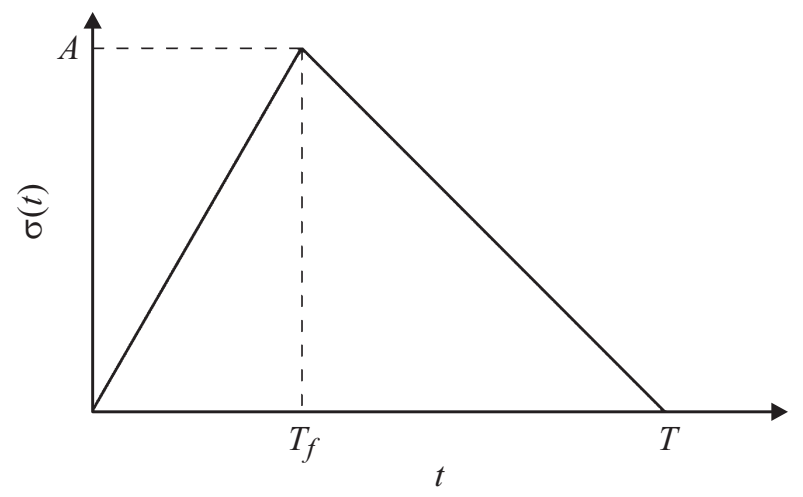

Рис. 6. Импульс напряжения. 
ческое выражения для $\sigma(t)$ в данном случае

$$
\begin{aligned}
\sigma(t) & =\frac{A t}{T_{f}}\left(\theta(t)-\theta\left(t-T_{f}\right)\right)+\frac{A}{T_{f}-T} \cdot(t-T) \\
& \times\left(\theta\left(t-T_{f}\right)-\theta(t-T)\right)
\end{aligned}
$$

где $\theta(t)$ - функция Хевисайда, $T$ - длительность импульса, $T_{f}$ - время роста импульса напряжения, $A-$ амплитуда исходного импульса нагружения. Далее будем считать, что разрыв кольцевого образца происходит на переднем фронте импульса напряжения, тогда

$$
\sigma(t)=\frac{A t}{T_{f}} \cdot \theta(t)
$$

Подставив выражение для напряжения (3) в критерий (2), получим следующую зависимость для предельного напряжения от времени разрушения:

$$
\begin{cases}\sigma^{*}=\frac{\sigma_{c}}{1-\frac{\tau}{2 t^{*}}}, & t^{*} \geqslant \tau \\ \sigma^{*}=\frac{2 \sigma_{c} \tau}{t^{*}}, & t^{*} \leqslant \tau .\end{cases}
$$

Здесь $\sigma^{*}$ - значение окружного напряжения в момент разрушения $t^{*}$.

При расчете инкубационного времени в качестве статической прочности для алюминия было принято $\sigma_{c}=110 \mathrm{MPa}$.

На рис. 5 сплошной линией показана зависимость прочности для алюминиевого образца от времени разрушения, полученная на основе системы (4). Инкубационное время также было вычислено из системы (4) и получилось равным $\tau=1.8 \mu \mathrm{s}$. Точками на рисунке обозначены экспериментальные данные.

Предложенный подход дает возможность моделировать поведение значений предельных напряжений различных материалов при высокоскоростных нагружениях. Инкубационное время, являющееся основой структурновременного подхода, позволяет моделировать поведение материалов при внешних воздействиях. Важным моментом является то, что инкубационное время не зависит от геометрии образца, а также от истории воздействия и является константой для рассматриваемого материала. В то же время данный подход предоставляет легкий и удобный способ для вычисления критических значений напряжений исследуемого материала.

\section{Заключение}

1. С использованием трех разработанных схем нагружения тонких алюминиевых кольцевых образцов магнитно-импульсным методом получены данные, на основе которых построена временная зависимость их прочности в широком диапазоне скоростей деформаций.

2. На основе структурно-временного подхода получена аналитическая зависимость предельных напряжений от времени разрушения, которая находится в хорошем соответствии с экспериментальными данными. Такой подход позволяет описать как ветвь кривой скоростного нагружения, так и ту ветвь, где процессы протекают квазистатически.

3. Определено инкубационное время разрушения для алюминиевых кольцевых образцов при магнитноимпульсных нагружениях. Полученный набор параметров $\left(\tau, \sigma_{c}\right)$ предоставляет возможность для моделирования процесса разрушения.

\section{Список литературы}

[1] Морозов В.А., Петров Ю.В., Лукин А.А., Кач В.М., Удовик А.Г., Атрошенко С.А., Грибанов Д.А., Федоровский Г.Д. // ЖТФ. 2011. Т. 81 Вып. 6. С. 51-56. [Morozov V.A., Petrov Yu.V., Lukin A.A. et al. // Tech. Phys. 2011. Vol. 56. N 6. P. 797-802.]

[2] Morozov V.A., Petrov Yu.V., Lukin A.A., Kats V.M., Atroshenko S.A., Fedorovskii G.D., Gribanov D.A., Zaichenko O.K. $13^{\text {th }}$ Intern. Conf. on Fracture (ICF 13), June 16-21, 2013, Beijing, China, ICF 13 Proceedings, Physical Aspects (s17-043).

[3] Морозов В.А., Петров Ю.В., Лукин А.А., Атрошенко С.А., Грибанов Д.А. // ЖТФ. 2014. Т. 84 Вып. 9. С. 78-85. [Morozov V.A., Petrov Yu.V., Lukin A.A. et al. // Tech. Phys. 2014. Vol. 59. N 9. P. 1338-1345.]

[4] Gunko Y.F., Zaychenko O.K., Lukin A.A., Morozov V.A., Gunko N.A. 2015 Intern. Conf. on Mechanics - Seventh Polyakhov's Reading 7106734.

[5] Zaychenko O.K., Lukin A.A., Morozov V.A. // Materials Physics and Mechanics. 2016. Vol. 26. N 1. P. 81-84.

[6] Петров Ю.В. // ДАН. 2004. Т. 395. № 5. С. 621-625. [Petrov Yu.V. // Doklady Physics. 2004. Vol. 49. N 4. P. 246 249.]

[7] Selyutina N., Petrov Y. // Procedia Structural Integrity. 2016. Vol. 2. P. 438-445.

[8] Златин Н.А., Пугачев Г.С., Мочалов С.М., Брагов А.М. // ФTT. 1975. Т. 17. Вып. 9. С. 2599-2602. [Zlatin N.A., Pugachev G.S., Mochalov S.M. et al. // Phys. Solid State. 1975. Vol. 17. N 9. P. 2599-2602.]

[9] Петров Ю.В. // ДАН. 2004. Т. 395. № 5. С. 621-625. [Petrov Y.V. // Dokl. Phys. 2004. Vol. 49. N 4. P. 246-249.]

[10] Петров Ю.В., Уткин А.А. // ЖТФ. 2015. Т. 85. Вып. 8. C. 58-62. [Petrov Yu.V., Utkin A.A. // Tech. Phys. 2015. Vol. 60. N 8. P. 1162-1166.]

[11] Михайлова Н.В., Волков Г.А., Мещеряков Ю.И., Петров Ю.В., Уткин А.А. // ЖТФ. 2017. Т. 87. Вып. 4. С. $527-$ 532. [Mikhailova N.V., Volkov G.A., Meshcheryakov Yu.I. et al. // Tech. Phys. 2017. Vol. 62. N 4. P. 547-552.]

[12] Петров Ю.В., Глебовский П.А. // ЖТФ. 2004. Т. 74 Вып. 11. С. 53-57. [Petrov Yu.V., Glebovskii P.A. // Tech. Phys. 2004. Vol. 49. N 11. P. 1447-1451.]

[13] Братов В.А., Петров Ю.В. // ДАН. 2007. Т. 416. № 5. C. 624-626. [Bratov V.A., Petrov Yu.V. // Dokl. Phys. 2007. Vol. 52. N 10. P. 565-567.] 